During the first 24 hours the temperature rose to $101^{\circ} \mathrm{F}$. and the pulse increased in proportion; the child was rather weak and restless. After this there was marked improvement, both temperature and pulse remaining normal except on the 20th, 21st, and 22nd, when there was a slight rise due to the child cutting a tooth. There was no vomiting; the bowels were opened naturally on the first day and regularly afterwards. During the first day small quantities of meat peptones and hot water were given by the mouth, and saline enemata; afterwards the meat peptones were continued and peptonised milk was given. On the 18th the wound was dressed; it looked very well and some of the stitches were removed. On the 25th the rest of the stitches were taken out and the wound was quite healed. The child went to the convalescent home on July 4th.

Remar $\bar{k} s$ by Mr. RoBInson.-Umbilical polypi are not infrequently met with on the site of the separated cord. Although sometimes ' in the past loosely spoken of as granulomata they rarely are so histologically. The majority show an adenomatous structure consisting of large Lieberkühn's crypts with goblet cells or of glands branched like those about the pyloric end of the stomach. Such a histological appearance suggests its origin-viz., from one of the epithelial-lined tracts in the cord ; these are two in number, the omphalo.mesenteric duct and the urachus.

In normal development the omphalo-mesenteric duct atrophies from the small intestine to the abdominal wall, setting free the bowel, and as a rule leaves behind no evidence of the site of its original attachment to the bowel. The commonest variation, probably, is for its central part to remain patent as Meckel's diverticulum, but it may, as in the present case, remain patent in its whole length. In other instances the lumen becomes obliterated and there will remain a ligamentous cord stretching from the bowel to the navel; the latter cord may again lose its connexion with the abdominal wall, remaining free or becoming abnormally adherent especially to the mesentery. These variations may, as is well known, play an important part in inducing different forms of intestinal obstruction. The urachus is the continuation of the bladder to the umbilical cord and related to the allantoic outgrowth from the hind-gut.

The histology of the polyp points to its origin from intestine, as in the recorded cases there is no account of one being composed of cells resembling those of the bladder; in this connexion, however, it must be borne in mind that a transition has here occurred from that resembling the lining of the hind-gut.

In nearly every case of a polyp the child is brought to the hospital with a history of discharge from the navel, and on examination the little raspberry-like growth is seen. It behoves the surgeon to examine every such case, most carefully inquiring as to, and seeing the character of, the discharge and looking for any lumen in the growth. When the discharge resembles the contents of the small intestine it is evident that there is a patent duct, the polyp in this instance being the prolapsed micous membrane. Having, then, established the existence of such a patency the surgeon must consider his position as to how and when to act. As to the former there can be no two opinions bearing in mind the serious complications which may arise from non-interference. The prospect of strangulation under or across the band must always loom largely before the surgeon until this has been made impossible by an operation. The surgeon must be guided when to act by the child's general condition and also by the amount of discharge from the opening. If this is small it may be assumed that the lumen of the duct is narrow and that, if the operation is postponed, no great developmental disproportion will ensue in the lumina of the two ends of the bowel; but if the discharge is free and what comes from the rectum is limited then he must bear in mind that there will probably be a disproportion in size which will be more marked the longer the operation is postponed.

As to the surgical technique employed in this case no further remarks are necessary, the general principles in vogue in abdominal surgery being adopted.

The literature of this subject is extremely scanty. Treves ${ }^{1}$ reports having successfully pérformed a plastic operation for the closure of the abnormal passage in a male infant a few weeks old, but he gives no details as to what that operation was. Beyond this case I can find no other recorded where

1 Intestinal Obstruetion, 1899, p. 42. such a plastic operation was deliberately done. From $\mathbf{m y}$ search several cases have been reported where in an operation for intestinal obstruction a band has been found, the remains of the duct with only its central part patent-a Meckel's diverticulum with a fibrous cord passing to the navel -in these the diverticulum and its continuation have been removed.

\section{ROYAL ISLE OF WIGHT COUNTY HOSPITAL.}

A CASE OF TRAUMATIC TETANUS ; RECOVERY.

(Under the care of Mr. F. J. WADHAM.)

THE value of antitoxin in the treatment of tetanus requires very careful examination. Numerous reports are published describing cases in which its administration has been followed by recovery, and a smaller number of cases in which death has resulted. Yet it must not be imagined that the numbers of the recorded cases represent the relative frequency of recovery and death after treatment with antitoxic serum. Before the introduction of the antitoxin a proportion, by no means small, of the cases of tetanus recovered, though statistics vary greatly as to the exact proportion, and it has not been definitely shown that the percentage of recoveries has been materially altered. In the present state of our knowledge the administration of the antitoxin should not preclude the use of other męans which have been found useful, such as the bromides. It would be well if we could obtain a long series of cases which had been treated in one institution both before and after the introduction of the antitoxin. We should then be in a better position to judge of its value. For the notes of the case we are indebted to Dr. R. C. H. Kennedy, house surgeon.

On Nov. 3rd, 1901, a man, aged 22 years, a groom, whilst working in a stable broke a pane of glass in the window and in so doing cut his right index finger on the dorsal surface of the proximal phalanx. He stated that the wound bled profusely and that in order to check the hæmorrhage he placed some cobweb on it. He did not suffer much pain. The wound suppurated slightly and then after a few days it closed up. On the 14th he felt some stiffness in his jaws and at dinner had difficulty in masticating his food and in swallowing. The wound broke down and he suffered some pain in it. On the 15th he consulted Mr. Wadham who immediately sent him to the Royal Isle of Wight County Hospital. He complained of pain and stiffness in the back and neck. Considerable trismus and risus sardonicus were present. The wound, which was about three-quarters of an inch long, was unhealthy-looking and discharging sanious pus. He was placed in a darkened room and put on bromide of potassium (30 grains every four hours), brandy (10 drachms every four hours), and beef-tea and milk (four ounces every two hours alternately). The wound was washed in a solution of perchloride of mercury (1 in 1000) and was dressed with a pad of gauze wrung out of a solution of the same strength. The axillary temperature was $98 \cdot 6^{\circ} \mathrm{F}$., the pulse was 60 , and the respirations were 20 . During the day the patient became very restless and all the tetanic symptoms became more marked 10 cubic centimetres of anti-tetanic serum were injected into the flank. The spasms now appeared to be more clonic in character than tonic, with exacerbations. The injection of serum was continued during the first seven days until 110 cubic cəntimetres had been given, not more than 10 cubic centimetres being given iat any one time. The various symptoms increased in severity till the fifth day after admission, when almost absolute trismus was present, swallowing was difficult, and great opisthotonos and pain in the back and neck with exacerba tion were caused by the slightest noise or movement. The passage of a catheter, which had been necessary since the second day, became very difficult owing to the spasm which it caused. Nitrite of amyl was now administered as an inhalation during the exacerbations and afforded marked relief.

The patient remained in this condition till Nov. 24th (twenty-first day after injury), when the exacerbations became less severe and less frequent, and diuresis became much increased. On the 26 th (twenty-third day) an enema was given, the bowels not having been opened since admission. On the 28 th bromism developed, the patient having taken is 
toto rather more than 42 drachms of bromide of pota-sium ; the bromide was stopped. On Dec. 3rd (thirtieth day) urine was passed naturally. The bowels were very loose and the patient was able to sit up in bed. On the 6th he was allowed bread and milk and custard in place of some of the fluid nourishment. 15 grains of bromide of potassium were given each night. On the 9th (thirty-sixth day) the patient was up for a couple of hours. There was no stiffness remaining except in the legs which were still somewhat rigid. On the 18th he had been up daily and was out on this day for some time. On the 30 th the patient was discharged quite recovered.

\section{Setlexical Socteties.}

\section{ROYAL MEDICAL AND CHIRURGICAL SOCIETY.}

Adjourned Discussion on Modern Methods of Vaccination and their Soientific Basis.

A MEeTING of this society was held on Jan. 28th, Dr. F. W. PAVY, the President, being in the chair.

Dr. SIDNEY CouplanD, in opening the adjourned discussion on Modern Methods of Yaccination and their Scientific Basis, said that the Act of 1898 had had a most beneticial effect on vaccination. It had greatly diminished the risk which resulted from vaccinal injury, since by making it obligatory to employ a glycerinated lymph nearly all the infective organisms had been eliminated. The performance of the operation at the child's home instead of at a station had reduced the accidents following vaccination to a minimum. He thought that the profession had not sufficiently considered the slighter injury which followed vaccination. In the majority of cases in which bad results followed it was not the lymph which was at fault but some accidental complication. He quite agreed with Dr. T. D. Acland that the Government should have full control of all the vaccine used in the country. With regard to what might be regarded as efficient vaccination he said that since the purified lymph might give rise to such a small amount of scarring the size and area of the scars could no longer be considered as evidence of effective vaccination, and reliance would have to be placed rather on the number of scars than on either their depth or area. It was a well-recognised fact that those persons with more scars were better protected than were those with fewer scars. With regard to the protective value which a previous attack of small-pox had, he said that out of 250 people who had had small-pox in childhood 10 contracted a second attack of small-pox when exposed to infection, and in an epidemic investigated by Dr. T. D. Savill an even larger proportion occurred-viz., nine out of 41 cases. With regard to the protective value of vaccination, he said that about one in four persons who had been vaccinated in infancy contracted small-pox if exposed to infection in later life. Revaccination conferred absolute immunity, and even in a patient who had had small-pox the immunity could be prolonged by revaccination. In children under 10 years of age who had been vaccinated in infancy the liability to contract small-pox when exposed to infection was one in 12 , while in those who were unvaccinated the liability was two in three. He did not consider that all even recently vaccinated persons were absolutely immune owing to their vaccination with various strains of lymph, some of which might have become inert.

Dr. A. E. Cope said that as a public vaccinator he wished to say a few words on the way in which the actual work of vaccination was carried out. He then gave details as to the cleansing of the arm, which he thought in the infart was best carried out with a little rectified spirit, while in revaceination a more thorough cleansing with 1 in 20 carbolic soap was advisable. With regard to the instrument used he was in favour of a platinum lancet with a hard iridium point-this could be sterilised easily in a flame without losing either its temper or its sharpness; it also possessed the further advantage that it did not get the dull, dirty appearance of a steel instrument after being heated in a flame. Before covering up the scratches he waited till an urticarial reaction had taken place and then covered the site of inoculation with a dresing of boric wool. One of the most troublesome complications which he had met with was the chronic eczema which sometimes started around the vaccination scars. He thonght that children should be revaccinater at 14 years of age, and that if this were done then the inconvenience of a swollen arm, which often resulted if the vaccination was left till the patient was between 30 and 35 years of age, would be avoided, for the effect would be less severe.

Mr. WILLIAM F. BLAKE asked what constituted successful vaccination. If two persons were vaecinated from the same tube and the one took but the other did not, could it be asserted that the person who did not take was protected against small-pox? He also asked whether it was possible to vaccinate an individual successfully with different strains of lymph within a period of a few months, as had been asserted by a speaker at a former meeting.

Dr. F. CHURCHILL asked if any age-limit could be fixed at which it was unnecessary to revaccinate adults. He had been in the habit of advising revaccination in persons even of advanced age.

Dr. BERnARD O'Connor said that he thought that revaccination should be performed at 14 years of age and that after that revaccination should be performed at fixed periods until the individual ceased to take.

Dr. F. St. George Mivart said that he had seen keloid ocour atter vaccination and that this had occurred in certain districts. He had been unable to discover any cause for this condition.

Dr. S. Monchton Copeman, in reply, said that the effect of glycerine in the lymph was to inhibit and eliminate all the non-spore-bearing organisms, and since most of the pathogenic organisms were non-spore-bearing the lymph was thus freed from all infective organisms. Even if such organisms as those of erysipelas and tubercle were intentionally added to the lymph in considerable quantities the subsequent addition of glycerine gradually eliminated them, so that after a given interval the lymph was free from any such infective qualities. He considered that the same amount of vaccination was necessary in the child as in the adult, and, as a rule, the vesiculation was less severe in relation to the amount of vaccine used in the child than in the adult. $\mathrm{He}$ said that no satisfactory method of standardising lymph had yet been discovered. There was no doubt that a person might contract small-pox twice or even three times. He referred to the great immunity which vaccination gave., and as an instance said that he had on two occasions accidentally inoculated himself from cases of confluent small-pox, with the result that no infection took place. With regard to inert lymph he said that when it was suspected that a vaccination had failed because of inert lymph the jatient should not at once be revaccinated but should wait a month, for a certain amount of local immunity seemed to be produced even by apparently inert lymph. It was generally assumed that the glycerinated lymph was less potent than was the older form of lymph. This he did not think was the case, for in the preparation of the calf lymph the epithelium in which the vaccine organisms were supposed to be was scraped off, whereas in the Jymph taken from the vesicles only the fluid portion was taken. In conclusion, he referred to the possibility of vaccination by internal administration.

\section{MEDICAL SOCIETY OF LONDON.}

\section{Discussion on the Nature and Treatment of Obesity.}

A MEETING of this society was held on Jan. 27th, Dr W. H. AllchiN, the President, being in the chair.

Sir T. LAUder Brunton opened a discussion on the Nature and Treatment of Obesity. He said that it was difficult to give an exact definition of obesity, but in general terms it might be said that obesity commenced when the patient's fat was obviously very much greater than normal or when it became inconvenient to himself. In order to define more accurately what he meant he gave the following figures. The average height of an Englishman was about five feet eight inches and his weight 11 stones. This remained pretty constant till the age of 40 years, when the weight generally began slightly to increase. According to van Noorden this weight might increase as much as 15 per cent. and still be within the normal limit. When it reached 30 per cent. the person was regarded as stout; from 30 per cent. to 35 per cent. as 